However, approximately one-third with PTSD have cognitive impairment regardless of exposure level.

\section{LEVERAGING MULTIPLE DATA SOURCES TO ASSIST OSHA IN ENFORCEMENT: EXAMINING ILLINOIS WORKERS' COMPENSATION DATA ON AMPUTATIONS}

Alison Krajewski, DC Dana Madigan, MPH, Linda Forst, MPH, Lee Friedman. University of Illinois at Chicago, Chicago, IL, USA

\subsection{6/oemed-2014-102362.214}

Objectives The US Occupational Safety and Health Administration (OSHA) relies on survey data to target high risk employers for safety inspections and enforcement. However, OSHA has limited resources and randomly targeting employers for safety inspections as a general strategy is inefficient. In this study, we explore the use of an alternative data source to assist OSHA with a more targeted inspection program.

Method We summarised Illinois Workers' Compensation Commission (IWCC) court filings for amputations from 2007 through 2012 and identified the industries and companies that are the top offenders for amputations in Illinois within this dataset.

Results In total, there were 1260 amputations, of which $27.1 \%$ were severe amputations (not restricted to digits). The industrial sectors with the highest proportion of severe amputations were the mining (50.0\%), government (40.6\%), and personnel/ employment services (temp employment) (40.0\%) sectors. The analysis identified a short list of companies and their corresponding worksites, comprising nearly $20 \%$ of the amputation claims filed through the court system.

Conclusions Using alternative datasets that provide a specific list of the worst offenders in a region can guide a more efficient use of OSHA resources and create a system that targets the companies with a poor safety record. Random inspections using industry level data from the US Bureau of Labour Statistics does not differentiate between companies that invest in safety within high risk industries from the poor actors. Workers' compensation data is only one resource among many that can be utilised to assist OSHA with targeted inspections of high risk companies.

\section{RECONSTRUCTION OF EXPOSED COHORT AND CUMULATIVE DOSE OF RADIO-CONTAMINATED BUILDING RESIDENTS FOR EPIDEMIOLOGICAL STUDY - UPDATED INFORMATION}

${ }^{1,2}$ Shu-O Chiang ${ }^{3}$ Chien-Hao Chen, ${ }^{4}$ Chuan-Jong Tung, ${ }^{5}$ Pesus Chou, ${ }^{2,6}{ }^{6}$ Jacqueline Whang-Peng, ${ }^{7}$ Hung-Yi Chiou, ${ }^{6}$ Chien-Jen Chen, ${ }^{6} Y_{i-H a u}$ Chen, ${ }^{8} \mathrm{Li}$-Hui Yu, ${ }^{2}$ Jang-Yang Chang, ${ }^{2}$ Lukas Jyuhn-Hsiarn Lee, ${ }^{1,2}$ Saou-Hsing Liou. ${ }^{1}$ Graduate Institute of Life Sciences, Academia Sinica, National Health Research Institutes and National Defense Medical Center, Taipei, Taiwan; ${ }^{2}$ Division of Environmental Health and Occupational Medicine, National Health Research Institutes, Miaoli, Taiwan; ${ }^{3}$ ESTAT Statistical Consulting Co., Ltd., Taipei, Taiwan; ${ }^{4}$ Department of Medical Imaging and Radiological Sciences, School of Medicine, Chang-Gang University, Taoyuan, Taiwan; ${ }^{5}$ Institute of Public Health, Yang-Ming University, Taipei, Taiwan; ${ }^{6}$ Academia Sinica, Taipei, Taiwan; ${ }^{7}$ School of Public Health, Taipei Medical University, Taipei, Taiwan; ${ }^{8}$ Department of Health, Taipei City Government, Taipei, Taiwan

10.1136/oemed-2014-102362.215

Objectives The purpose of this study is to reconstruct of RCB cohort and to survey occupancy factors for estimation of cumulative dose in study of health risk of Radio-contaminated building (RCB) occupants.

Method The cohort of RCB occupants was established based on household, school and labour registration. Survey of current and retrospective occupancy factors in each age stratum was conducted to estimate the cumulative dose. Risk-period adjusted cumulative dose was generated in this study.

Results There were totally 32622 in this study cohort, including 13120 residents of RCBs, 15368 students in RCB schools, and 4328 labours in RCB business workplaces. The mean and maximal dose in the year of RCB built was 32.7 and 998.56 $\mathrm{mSv}$, respectively (Table 2 ). The main difference between retrospective (RCB residents) and current (general population) occupancy factors was RCB residents in the past had 2 more hours in living room, while current general population had 2 more hours in bed room. When bedroom was subdivided into bed and desk, we found current general population had 2 more hours in the desk of bed room. In total, the mean cumulative dose was $7.06 \mathrm{mSv}$ with range of $0-1751 \mathrm{mSv}$. The mean cumulative dose for residents, labours, and students were 16.08, 2.91, and 0.48 $\mathrm{mSv}$, respectively.

Conclusions We have established a new RCB cohort and provided new information of cumulative dose estimation. These data could provide valid information on the risk of cancers and non-cancer diseases at low dose and low dose-rate radiation exposure.

\section{WORK ORGANISATIONAL CHARACTERISTICS AND PSYCHOLOGICAL DISTRESS IN FRENCH CALL-CENTRES. IS THERE A DIRECT EFFECT?}

Stephanie Boini, Dominique Chouaniere, Pascal Wild. Occupational Health and Safety Institute (INRS), Vandoeuvre-Les-Nancy, France

\subsection{6/oemed-2014-102362.216}

Objectives In cross sectional studies, relationships between perceived stressors and self-reported health are suspected of monomethod bias related to negative affectivity. The purpose of this work was to test if work organisational characteristics (WOC) reported by managers were related to psychological distress (PD) reported by call-handlers, taking into account their perception of work conditions (perceived strain) and usual individual confounders.

Method Managers of 107 call-centres were queried by occupational physicians on their call-centres' WOC. 4002 call-handlers (2,929 women were considered here) of these call-centres completed self-reported questionnaires in order to evaluate perceived work strain (Karasek and Siegrist questionnaires) and PD (12items General Health Questionnaire). A 2-level analysis tested the relationships between 14 WOC and PD score, with and without adding perceived strain as confounder, assuming that unchanged coefficients between WOC and PD score flag a direct effect of WOC on psychological distress. We also analysed perceived strain as an effect modificator for WOC using interactions.

Results Five of 14 WOC were significantly related to the PD score: type of calls, call-centre size, number of activity parameters displayed on screen, instructions for client relationships, and required control role of supervisor. In adding perceived strain, the relationship only remained for the type of call.

Conclusions Main results of this study are (1) existence of relationships between WOC reported by managers and psychological distress reported by call-handlers (2) most of effects of WOC are moderated by the perception of work strain. 